LM-06K051

May 9, 2006

\title{
Determination of Thermal Conductivity from a Surface Temperature Measurement: Theory for the $3 \omega$ Method
}

Y Mikata

\section{NOTICE}

This report was prepared as an account of work sponsored by the United States Government. Neither the United States, nor the United States Department of Energy, nor any of their employees, nor any of their contractors, subcontractors, or their employees, makes any warranty, express or implied, or assumes any legal liability or responsibility for the accuracy, completeness or usefulness of any information, apparatus, product or process disclosed, or represents that its use would not infringe privately owned rights. 


\title{
Determination of Thermal Conductivity from a Surface Temperature Measurement: Theory for the $3 \omega$ Method
}

\author{
Y. Mikata \\ Lockheed Martin \\ P.O. Box 1072 \\ Schenectady, NY 12301-1072
}

\section{Introduction}

In order to measure thermal conductivity of a material, a so-called $3 \omega$ method [1-5] is sometimes used. The key theoretical framework for the $3 \omega$ method is the determination of a steady-state time-harmonic temperature variation of the solid, whose thermal conductivity is to be measured, when the solid is heated by a time-harmonic heat source. Even though there have been a number of papers published on the $3 \omega$ method [2-5], none of them have provided an exact closed form solution for the steady-state time-harmonic temperature variation of the solid in a form which can lead to an exact closed form expression for the temperature variation amplitude and the phase shift. In this paper, an exact closed form solution is developed for a 1D heat conduction problem with a time-harmonic heat flux. This is a mathematical model for a thermal conductivity measurement for a thin film.

\section{Problem definition}

As a mathematical model for a thermal conductivity measurement, let us consider a 1D heat conduction problem as shown below.

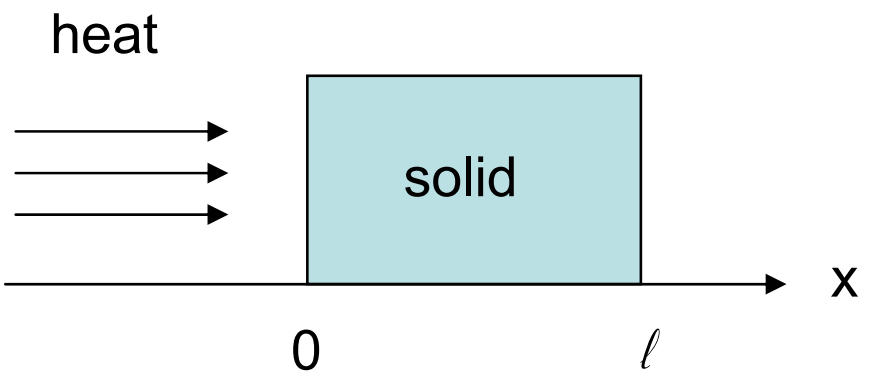

Fig. 1. Schematic of 1D heat conduction problem 
In a thermal conductivity experiment using the $3 \omega$ method, the solid is heated up by an electric AC heater attached at the surface, and the solid temperature rises. But eventually the solid temperature becomes steady state. The heat generated by the AC heater is modeled as a time-harmonic heat flux at $x=0$. By measuring the resulting time-harmonic temperature in the solid, the thermal conductivity of the solid can be determined. The 1D heat conduction problem shown above is very similar to the heat conduction problem associated with a photo-acoustic measurement [6] for obtaining the thermal conductivity. The main difference is that, in the photo-acoustic measurement, usually the heat conduction problem is defined for a multilayer system. The extension to a multilayer system for our problem is relatively straightforward. For our system, the governing equation for the heat conduction in the solid is given by

$$
\frac{1}{\alpha_{s}} \frac{\partial \phi}{\partial t}=\frac{\partial^{2} \phi}{\partial x^{2}} \quad 0 \leq x \leq l
$$

where $\alpha_{s}$ is the thermal diffusivity of the solid given by

$$
\alpha_{s}=\frac{k}{\rho c_{p}}
$$

Here $\rho$ is the density, and $c_{p}$ is the specific heat of the material. In Eq. (1), $\phi$ is a temperature difference, and is defined as

$$
\phi=\mathrm{T}_{\mathrm{s}}-\mathrm{T}_{0}
$$

where $T_{s}$ is a temperature in the solid, and $T_{0}$ is an ambient temperature. The boundary conditions are given by

$$
\begin{aligned}
& -k \frac{\partial \phi}{\partial x}(0, t)=q(1-\cos \omega t) \quad(q>0) \\
& \phi(l, t)=0
\end{aligned}
$$

where $q$ is the heat flux in the positive $x$-direction (into the solid). Since the heat is put into the solid in our problem, q is positive. The solution to (1) and (4) can be expressed as

$$
\phi(x, t)=\operatorname{Re}\left[\phi_{1}(x, t)\right]-\frac{q}{k}(x-l)
$$

where $\operatorname{Re}\left[\right.$ ] denotes the real part, and $\phi_{1}(x, t)$ is the solution to the following equations.

$$
\frac{1}{\alpha_{s}} \frac{\partial \phi_{1}}{\partial t}=\frac{\partial^{2} \phi_{1}}{\partial x^{2}} \quad 0 \leq x \leq l
$$




$$
\begin{aligned}
& -k \frac{\partial \phi_{1}}{\partial x}(0, t)=-q e^{i \omega t} \\
& \phi_{1}(l, t)=0
\end{aligned}
$$

Since our goal is to obtain the solution as $t \rightarrow \infty$, initial conditions are not needed. Eqs. (6), (7) and (8) define the boundary value problem for our $1 \mathrm{D}$ heat conduction problem.

\section{Mathematical formulation}

Let us set

$$
\phi_{1}(x, t)=\Phi_{1}(x) e^{i \omega t}
$$

Here it should be noted that $\phi_{1}(\mathrm{x}, \mathrm{t})$ as well as $\Phi_{1}(\mathrm{x})$ are both complex functions. Substituting (9) into (6), we obtain

$$
\frac{d^{2} \Phi_{1}}{d x^{2}}-\frac{i \omega}{\alpha_{s}} \Phi_{1}=0
$$

Substituting (9) into (7) and (8), we obtain

$$
\begin{aligned}
& -k \frac{d \Phi_{1}}{d x}(0)=-q \\
& \Phi_{1}(l)=0
\end{aligned}
$$

Solving (10), we obtain

$$
\Phi_{1}(x)=S_{1} e^{\lambda x}+S_{2} e^{-\lambda x}
$$

where $S_{1}$ and $S_{2}$ are the unknown constants to be determined from the boundary conditions (11), and

$$
\lambda=\sqrt{\frac{i \omega}{\alpha_{s}}}=\frac{1+i}{\sqrt{2}} \sqrt{\frac{\omega}{\alpha_{s}}}=\sqrt{\frac{\omega}{2 \alpha_{s}}}(1+i)
$$

Substituting (12) into (11), and solving the resulting linear algebraic equation, we obtain

$$
S_{1}=\frac{q}{k \lambda} \frac{e^{-\lambda l}}{D}, \quad S_{2}=-\frac{q}{k \lambda} \frac{e^{\lambda l}}{D}
$$


where

$$
D=e^{\lambda l}+e^{-\lambda l}
$$

Substituting (14) into (12), we have

$$
\Phi_{1}(x)=\frac{q}{k \lambda D}\left[e^{\lambda(x-l)}-e^{-\lambda(x-l)}\right]
$$

From (13) and (15), we have

$$
\begin{aligned}
& \frac{1}{\lambda}=\frac{1}{\sqrt{\frac{\omega}{2 \alpha_{s}}(1+i)}}=\frac{1-i}{2} \sqrt{\frac{2 \alpha_{s}}{\omega}}=\sqrt{\frac{\alpha_{s}}{2 \omega}}(1-i) \\
& \frac{1}{D}=\frac{1}{e^{z(1+i)}+e^{-z(1+i)}}=\frac{1}{e^{z}(\cos z+i \sin z)+e^{-z}(\cos z-i \sin z)} \\
& =\frac{1}{\cos z\left(e^{z}+e^{-z}\right)+i \sin z\left(e^{z}-e^{-z}\right)}=\frac{\cos z\left(e^{z}+e^{-z}\right)-i \sin z\left(e^{z}-e^{-z}\right)}{e^{2 z}+e^{-2 z}+2 \cos 2 z} \\
& =\frac{\cos z \cosh z-i \sin z \sinh z}{\cos 2 z+\cosh 2 z}
\end{aligned}
$$

where $\mathrm{z}$ is a non-dimensional parameter defined by

$$
z=\sqrt{\frac{\omega}{2 \alpha_{s}} l}
$$

Substituting (17) into (16), we obtain

$$
\begin{aligned}
& \Phi_{1}(x)=\frac{q}{k} \sqrt{\frac{\alpha_{s}}{2 \omega}}(1-i) \frac{1}{D}\left[e^{-\lambda l\left(1-\frac{x}{l}\right)}-e^{\lambda l\left(1-\frac{x}{l}\right)}\right] \\
& =\frac{q}{k} \sqrt{\frac{\alpha_{s}}{2 \omega}}(1-i) \frac{1}{D}\left[e^{-z\left(1-\frac{x}{l}\right)(1+i)}-e^{z\left(1-\frac{x}{l}\right)(1+i)}\right] \equiv \frac{q}{k} \sqrt{\frac{\alpha_{s}}{2 \omega}} \frac{P}{a}
\end{aligned}
$$

where

$$
a=\cos 2 z+\cosh 2 z
$$




$$
P=(1-i)(\cos z \cosh z-i \sin z \sinh z)\left[e^{-z\left(1-\frac{x}{l}\right)(1+i)}-e^{z\left(1-\frac{x}{l}\right)(1+i)}\right]
$$

Let us set

$$
y=z\left(1-\frac{x}{l}\right)
$$

From (20-2), we have

$$
P=-2(1-i)\left(P_{1}+i P_{2}\right)=-2 \sqrt{2} e^{-i \frac{\pi}{4}} \sqrt{P_{1}^{2}+P_{2}^{2}}(\cos \delta+i \sin \delta)
$$

where

$$
\begin{aligned}
& P_{1}=\cos z \cosh z \cos y \sinh y+\sin z \sinh z \sin y \cosh y \\
& P_{2}=\cos z \cosh z \sin y \cosh y-\sin z \sinh z \cos y \sinh y \\
& \cos \delta=\frac{P_{1}}{\sqrt{P_{1}^{2}+P_{2}^{2}}}, \quad \quad \sin \delta=\frac{P_{2}}{\sqrt{P_{1}^{2}+P_{2}^{2}}}
\end{aligned}
$$

From (23), we have

$$
P_{1}^{2}+P_{2}^{2}=\frac{1}{4}(\cosh 2 z+\cos 2 z)(\cosh 2 y-\cos 2 y)
$$

Substituting (25) into (22), we obtain

$$
P=-\sqrt{2} \sqrt{(\cosh 2 z+\cos 2 z)(\cosh 2 y-\cos 2 y)} e^{i\left(\delta-\frac{\pi}{4}\right)}
$$

Substitution of (26) into (19) yields

$$
\Phi_{1}(x, t)=-\frac{q}{k} \sqrt{\frac{\alpha_{s}}{\omega}} \sqrt{\frac{\cosh 2 y-\cos 2 y}{\cosh 2 z+\cos 2 z}} e^{i\left(\delta-\frac{\pi}{4}\right)}
$$

where (20-1) has been used. From (27), (9) and (5), we finally obtain

$$
\phi(x, t)=-\frac{q}{k} \sqrt{\frac{\alpha_{s}}{\omega}} \sqrt{\frac{\cosh 2 y-\cos 2 y}{\cosh 2 z+\cos 2 z}} \cos (\omega t-\beta)+\frac{q l}{k}\left(1-\frac{x}{l}\right)
$$

where 


$$
\beta=\frac{\pi}{4}-\delta
$$

From (23) and (24), we have

$$
\begin{aligned}
\cos \delta & =\frac{2(\cos z \cosh z \cos y \sinh y+\sin z \sinh z \sin y \cosh y)}{\sqrt{(\cosh 2 z+\cos 2 z)(\cosh 2 y-\cos 2 y)}} \\
\sin \delta & =\frac{2(\cos z \cosh z \sin y \cosh y-\sin z \sinh z \cos y \sinh y)}{\sqrt{(\cosh 2 z+\cos 2 z)(\cosh 2 y-\cos 2 y)}}
\end{aligned}
$$

Eq. (28) is the solution to our original problem. Eq. (28) can be rewritten as

$$
\phi(x, t)=-C \cos (\omega t-\beta)+\frac{q l}{k}\left(1-\frac{x}{l}\right)
$$

where the amplitude $C(>0)$ is defined by

$$
C=\frac{q}{k} \sqrt{\frac{\alpha_{s}}{\omega}} \sqrt{\frac{\cosh 2 y-\cos 2 y}{\cosh 2 z+\cos 2 z}}
$$

Eq. (21) can be rewritten as

$$
y=m z, \quad \text { where } \quad m=1-\frac{x}{l}
$$

Since $0 \leq x \leq 1$, we have from (33)

$$
0 \leq \mathrm{m} \leq 1
$$

From (18), we have

$$
\sqrt{\frac{\alpha_{s}}{\omega}}=\frac{l}{\sqrt{2} z}
$$

Substituting (33) and (35) into (32), we have

$$
C=\frac{q l}{\sqrt{2} k} \frac{1}{z} \sqrt{\frac{\cosh 2 m z-\cos 2 m z}{\cosh 2 z+\cos 2 z}}
$$

From (35) and (36), we have 


$$
\lim _{\omega \rightarrow \infty} C=\lim _{z \rightarrow \infty} C=0
$$

Therefore, the temperature difference amplitude $C$ goes to zero as the angular frequency $\omega$ goes to infinity everywhere. However, the amplitude drop would be more significant in the inside of the material $(0<x \leq \ell, 0 \leq m<1)$ than at the surface $(x=0, m$ $=1$ ) because of the negative exponential term which comes out of cosh function inside the square root in Eq. (36). For the phase shift angle, $\beta$, the high frequency limit does not exist in the inside of the material. However, at the surface $(m=1)$, there is a nice high frequency limit as will be shown in the following.

From (28), we have

$$
\phi(0, t)=-\frac{q}{k} \sqrt{\frac{\alpha_{s}}{\omega}} \sqrt{\frac{\cosh 2 z-\cos 2 z}{\cosh 2 z+\cos 2 z}} \cos (\omega t-\beta)+\frac{q l}{k}
$$

where $\beta$ is defined by (29), and

$$
\begin{array}{ll}
\cos \delta=\frac{\sinh 2 z}{\sqrt{\cosh ^{2} 2 z-\cos ^{2} 2 z}} & \text { at } \mathrm{x}=0 \\
\sin \delta=\frac{\sin 2 z}{\sqrt{\cosh ^{2} 2 z-\cos ^{2} 2 z}} &
\end{array}
$$

From (39), we obtain

$$
\tan \delta=\frac{\sin 2 z}{\sinh 2 z} \quad \text { at } \mathrm{x}=0
$$

It can be shown from (29) and (39) that $0 \leq \beta<\pi / 2$. From (29), (38) and (39), we also obtain

$$
\begin{array}{lll}
\lim _{z \rightarrow 0} \delta(z)=\frac{\pi}{4} & \lim _{z \rightarrow 0} \beta(z)=0 & \text { at } \mathrm{x}=0 \\
\lim _{z \rightarrow \infty} \delta(z)=0, & \lim _{z \rightarrow \infty} \beta(z)=\frac{\pi}{4} & \text { at } \mathrm{x}=0 \\
\lim _{\omega \rightarrow \infty} \phi(0, t)=\frac{q}{k_{s}} \sqrt{\frac{\alpha_{s}}{\omega}} \cos \left(\omega t-\frac{\pi}{4}\right)+\frac{q l}{k_{s}} &
\end{array}
$$

The above expression (41-3) gives the asymptotic limit of the surface temperature difference as the angular frequency $\omega \rightarrow \infty$. It can be also shown from (28) that after some algebraic manipulations 


$$
\lim _{\omega \rightarrow 0} \phi(x, t)=0
$$

which is consistent with the definition of the boundary value problem defined by (1) and (4), when $\omega=0$.

\section{Numerical results and discussion}

Let us first rewrite (38) as

$$
\phi(0, t)=-F \cos (\omega t-\beta)+\frac{q l}{k}
$$

where the temperature difference amplitude $F(>0)$ is given by

$$
F=\frac{q l}{\sqrt{2} k} \frac{1}{z} \sqrt{\frac{\cosh 2 z-\cos 2 z}{\cosh 2 z+\cos 2 z}}
$$

The phase shift angle $\beta$ is defined by (29), and $\delta$ in (29) is defined by (39) when $x=0$. $z$ is defined by (18), which is repeated below for completeness.

$$
z=\sqrt{\frac{\omega}{2 \alpha_{s}} l}
$$

From (44), a non-dimensional temperature difference amplitude $f(z)$ can be defined as

$$
f(z) \equiv \frac{F}{\frac{q l}{\sqrt{2} k}}=\frac{1}{z} \sqrt{\frac{\cosh 2 z-\cos 2 z}{\cosh 2 z+\cos 2 z}}
$$

Since we already know that $0 \leq \beta<\pi / 2$ from the previous section, the phase shift angle $\beta$ can be uniquely determined by

$$
\beta=\frac{\pi}{4}-\tan ^{-1}\left[\frac{\sin 2 z}{\sinh 2 z}\right] \quad(0 \leq \beta<\pi / 2)
$$

The numerical results for the non-dimensional temperature difference amplitude $f(z)$ and the phase shift angle $\beta$ as a function of $z$ are shown in Fig.2, and Fig. 3, respectively. 


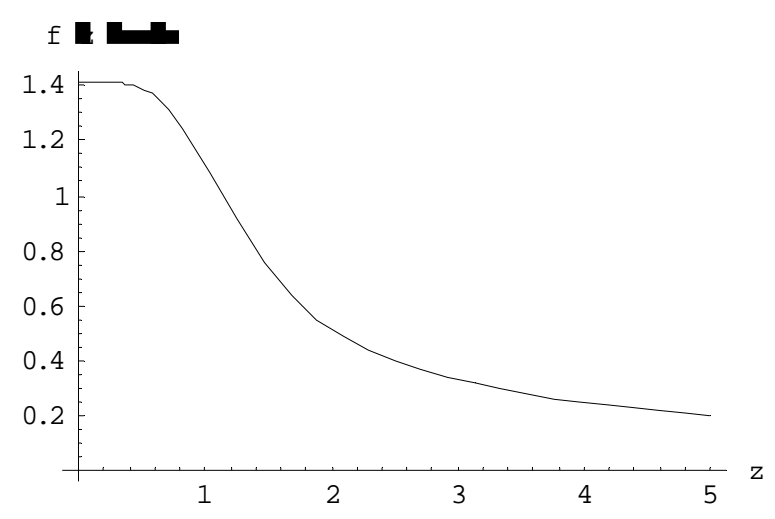

Fig.2. Non-dimensional temperature difference amplitude $f(z)$

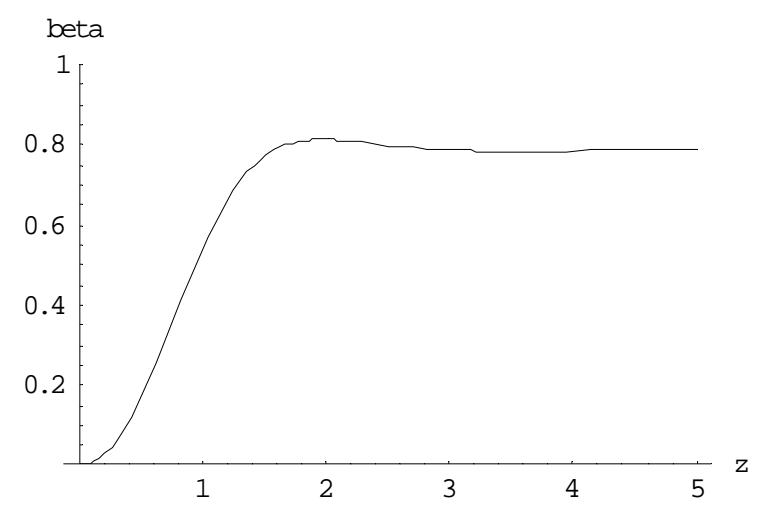

Fig.3. Phase shift angle $\beta$

It should be noted from (45), (46), and Figs. 2 and 3 that

$$
\begin{aligned}
& \lim _{z \rightarrow 0} f(z)=\sqrt{2} \\
& \lim _{z \rightarrow \infty} f(z)=0 \\
& \lim _{z \rightarrow 0} \beta=0 \\
& \lim _{z \rightarrow \infty} \beta=\frac{\pi}{4}
\end{aligned}
$$

It can be also shown from (61) that

$$
\underset{z>0}{\operatorname{Max}} \beta(z)=0.813266 \quad \text { at } z=1.9633
$$

Therefore, the experimentally useful range of $z$ for the phase shift angle measurement is $0<z<z_{\max }(=1.9633)$. 


\section{Determination of the thermal conductivity and the thermal diffusivity from a surface temperature measurement}

Suppose that we obtain $F$ and $\beta$ (and therefore $\delta$ ) from a measurement for a given heat flux $\mathrm{q}$, a given angular frequency $\omega$, and a given length $/$ of the specimen. Then the thermal conductivity $k$ and the thermal diffusivity $\alpha_{s}$ can be determined as follows.

1. Solve (40) for $z$ knowing the value of $\delta$. Since this is a transcendental equation, it has to be solved numerically.

2. Once $z$ is determined, the thermal diffusivity $\alpha_{s}$ can be determined from (18).

3. Once $z$ is determined, the thermal conductivity $k$ can be determined from (44) using the value of $F$.

From (2), we have

$$
\rho c_{p}=\frac{k}{\alpha_{s}}
$$

Thus the heat capacity $\rho c_{p}$ can be also obtained. If we know the density $\rho$, we can thus determine the specific heat $c_{p}$.

On the other hand, if we already know the heat capacity $\rho c_{p}$, then we can determine the thermal conductivity $k$ from (50), knowing the value of $\alpha_{s}$ after the step 2 above. The thermal conductivity thus determined can be compared to the thermal conductivity obtained from the step 3 above, and therefore, it can be used as a consistency check of the experimental data.

\section{Conclusion}

This paper solves a heat conduction problem associated with a $3 \omega$ method for obtaining the thermal conductivity of a solid. The thermal conductivity measurement is modeled as a $1 \mathrm{D}$ heat conduction problem, and the AC current heat source is modeled as a time-harmonic heat flux at the boundary of the solid. An exact closed form solution is obtained for this boundary value problem, which could be used with experimental data obtained from the $3 \omega$ method to determine the thermal conductivity of a solid. Also this exact solution may be used as a bench mark solution for a numerical solution, or other approximate solutions. 


\section{Acknowledgement}

The help from Dr. Dale Taylor, Lockheed Martin, Schenectady, NY, for the author's understanding of $3 \omega$ method is greatly appreciated.

\section{References}

1. O.M. Corbino, Z. Physik, vol. 11, 413 (1910).

2. L.R. Holland, J. Appl. Phys. Vol 34, pp. 2350-2357 (1963).

3. D.G. Cahill, "Thermal conductivity measurement from 30 to $750 \mathrm{~K}$ : the $3 \omega$ method," Rev. Sci. Instrum. Vol. 61, pp. 802-808 (1990).

4. S.M. Lee, and D.G. Cahill, "Heat transport in thin dielectric films," J. Appl. Phys. Vol. 81, pp. 2590-2595 (1997).

5. L. Lu, W. Yi, and D.L. Zhang, "A $3 \omega$ method for specific heat and thermal conductivity measurements," Rev. Sci. Instrum. Vol. 72, pp. 2996-3003 (2001).

6. A. Rosencwaig and A. Gersho, "Theory of the photoacoustic effect with solids," J. Appl. Phys. Vol. 47, pp. 64-69. 